\title{
Psychological mechanisms associated with food security status and BMI in adults: a mixed methods study
}

\author{
Candice A Myers* ${ }^{\circ}$, Robbie A Beyl, Corby K Martin, Stephanie T Broyles and \\ Peter T Katzmarzyk \\ Pennington Biomedical Research Center, Baton Rouge, LA 70808, USA
}

Submitted 7 August 2019: Final revision received 10 February 2020: Accepted 6 March 2020: First published online 29 June 2020

\begin{abstract}
Objective: This study examined psychological constructs (delay discounting, grit, future time perspective and subjective social status) in relation to food security status and body weight.

Design: A simultaneous triangulation mixed methods design was used to collect quantitative and qualitative data. Quantitative data were collected in fifty-six adults. Independent variables included food security status (food secure or food insecure) and BMI category (normal weight or overweight/obese). Participants, matched on race (African American and White), were categorised into four food security status by BMI category groups. Psychological constructs were measured via validated questionnaires. Qualitative data were collected in a subsample of twelve participants via in-depth interviews.

Setting: This study was conducted in Baton Rouge, Louisiana.

Participants: The sample was $66 \%$ female and $48 \%$ African American with a mean age of $32.3(\mathrm{SD} 9.2)$ years and BMI of $28.8(\mathrm{SD} 7.7) \mathrm{kg} / \mathrm{m}^{2}$.

Results: Quantitative results showed that food-insecure participants with overweight/ obesity had greater delay discounting $(-3.78 v .-6 \cdot 16, P=0.01 ;-3.78 v .-5.75$, $P=0.02)$ and poorer grit $(3.37 v .3 .99, P=0.02 ; 3.37 v .4 .02, P=0.02)$ than their food-secure counterparts and food-insecure participants with normal weight. Food-insecure participants with overweight/obesity also had a shorter time period for financial planning $(0.72 v \cdot 4 \cdot 14, P=0.02)$ than food-secure participants with normal weight. Qualitative data largely supported quantitative findings with participants discussing varied perceptions of psychological constructs.

Conclusions: This study found differences in delaying gratification, grit and financial planning between food security status and body weight groups.
\end{abstract}

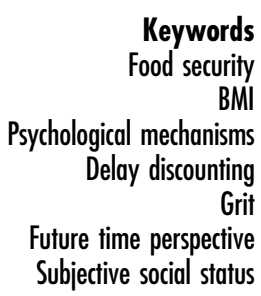

Food insecurity, or the limited or uncertain 'availability of nutritionally adequate and safe foods or the ability to acquire acceptable foods in socially acceptable ways ${ }^{(1)}$, is a pressing health issue given its association with greater body weight ${ }^{(2-4)}$. Most research evidence has implicated diet and eating behaviours in the linkage between food insecurity and obesity, including poor diet quality and maladaptive dietary behaviours ${ }^{(5-9)}$, as well as physiological adaptations to episodes of food insecurity ${ }^{(5,10-12)}$. While this evidence demonstrates that food insecurity is a risk factor for obesity and related comorbidities (e.g. metabolic syndrome and poor cardiovascular health) ${ }^{(13,14)}$, more research is still needed to better identify potential mechanisms that work in this pathway.
A recent body of narrative literature has developed new hypotheses to elucidate causal pathways in the relationship between food insecurity and greater body weight ${ }^{(2,15,16)}$. Nettle et al. ${ }^{(2)}$ propose the 'insurance hypothesis' to explain why food insecurity is a driver of obesity. They postulate that decision-making psychological mechanisms are affected by perceived food insecurity to influence food consumption choices. Specifically, in a food-insecure environment, these psychological mechanisms influence decisions that result in consuming greater amounts of food, which can lead to increased body weight. Similarly, Dhurandhar focused on the role of social status in developing the 'resource scarcity hypothesis' to explain how food insecurity can lead to increased body weight. In this case, 
food insecurity coupled with perceived low social status as a psychosocial stressor can result in excess energy intake and, in turn, body weight gain ${ }^{(15)}$.

A number of studies have examined how psychological mechanisms relate to body weight. Constructs such as delay discounting, less grit (perseverance) and lack of future time orientation are each associated with greater body weight ${ }^{(17-19)}$. Delay discounting is the inability to delay immediate gratification and a preference towards immediate, smaller rewards rather than delayed, larger rewards ${ }^{(20)}$. Grit is conceptualised as perseverance and passion for long-term goals ${ }^{(21)}$, while future time perspective captures the ability to prospectively think about the future $^{(18)}$. Importantly, few studies have explored these psychological mechanisms in food-insecure samples. One study demonstrated the association of delay discounting and poor future time orientation with food insecurity ${ }^{(22)}$. Another study found that households with parents that reported greater grit were less likely to report being food insecure ${ }^{(23)}$. However, these studies did not triangulate these associations with body weight. Further evidence also shows that greater subjective social status is associated with lower body weight ${ }^{(24)}$ and perceived social status mediates the relationship between food insecurity and obesity ${ }^{(25)}$

Food insecurity may play a significant role in the aetiology of obesity. Yet, more evidence is needed to better explicate potential mechanisms that underlie this association. The objective of this exploratory study was to investigate the role of four psychological mechanisms in the relationship between food insecurity and obesity. To the authors' knowledge, this is the first study to assess four key psychological constructs - delay discounting, grit, future time perspective and subjective social status - in a sample of food-secure and food-insecure adults with and without obesity. This objective was achieved via a mixed methods design collecting both quantitative and qualitative data. A mixed methods approach was undertaken to triangulate quantitative results with in-depth qualitative data to lend a more comprehensive investigation of the interplay between food security status, body weight and multiple decision-making psychological mechanisms.

\section{Methods}

\section{Study design and participants}

This study used a simultaneous triangulation mixed methods design to collect different, but complementary, types of data ${ }^{(26)}$. Specifically, a triangulation framework was used to collect (1) quantitative data from a sample of participants and (2) qualitative data from a subsample of those participants. Quantitative and qualitative data collection occurred concurrently, and data analysis occurred after all data collection was completed ${ }^{(27)}$. For each type of data (quantitative and qualitative), appropriate analytical procedures were undertaken ${ }^{(28)}$. Given this, quantitative and qualitative findings are presented separately, but comparisons between the two types of findings were made to integrate results.

Participants were recruited from the Baton Rouge, Louisiana community. Recruitment methods included email blasts, social media posts and in-person announcements by study staff at community events. Additionally, in partnership with a local food pantry serving a predominantly low-income neighbourhood, participants were recruited on-site while queuing for their food pantry visit via flyers, word of mouth from food pantry volunteers and clients, and in-person by study staff. This community partnership further allowed for some study visits to occur on-site at the food pantry as well.

A convenience sample of fifty-six food-secure and food-insecure women and men was recruited into the study. Potential participants were screened to assess eligibility. Eligible participants had a BMI of $20 \mathrm{~kg} / \mathrm{m}^{2}$ or greater, were aged 18-49 years, spoke English and were not currently pregnant. Food insecurity is associated with malnutrition, including both undernutrition (underweight) and overnutrition (overweight and obesity) ${ }^{(10,29)}$, the latter of which was the focus of this study. While many studies use a BMI of $<18.5 \mathrm{~kg} / \mathrm{m}^{2}$ as the typical cut-off indicating underweight, this study opted for a more conservative cut-off of BMI $20 \mathrm{~kg} / \mathrm{m}^{2}$ in order to avoid the potential association between food insecurity and undernutrition/underweight and better focus on the association between food insecurity and overnutrition/ overweight and obesity. Additionally, the age range of eligible participants was restricted to 18-49 years to focus on young and middle-age adults and avoid potential age-related changes in physiological, psychological and social factors ${ }^{(30)}$. Eligible participants provided written informed consent and then completed all quantitative study assessments.

\section{Quantitative data collection}

Quantitative data from fifty-six participants were collected via a basic anthropometric assessment measuring only height and weight, multiple questionnaires and a sociodemographic survey. Participants completed paper or electronic versions of the questionnaires and survey. Before completing these, health literacy was assessed using the Rapid Estimate of Adult Literacy in Medicine short form. The Rapid Estimate of Adult Literacy in Medicine short form is a standardised series of seven health-related words that participants were asked to read aloud ${ }^{(31)}$. A score of 6 or less indicates reading comprehension below high school level (low health literacy), while a score of 7 indicates reading comprehension at the high school level. Participants who scored 0-3 ( $n$ 3) were provided assistance by study staff in completing the questionnaires and survey. Patients who scored 4-7 ( $n$ 53) were allowed to complete 
the questionnaires and survey on their own, but were offered assistance if needed.

All quantitative data were collected and managed electronically via the Research Electronic Data Capture system $^{(32,33)}$. Research Electronic Data Capture is a secure, web-based software platform designed to support data capture for research studies, providing (1) an intuitive interface for validated data capture; (2) audit trails for tracking data manipulation and export procedures; (3) automated export procedures for seamless data downloads to common statistical packages and (4) procedures for data integration and interoperability with external sources.

\section{Independent variables}

Food security status was measured using the Six-Item Food Security Survey Module, which is a well-validated and widely used questionnaire ${ }^{34,35)}$. The six-item module was developed by researchers at the National Center for Health Statistics as an effective short form of the eighteen-item scale ${ }^{(36)}$. This questionnaire references reducing meal size or skipping meals, not having enough money for food, experiencing hunger and the lack of food availability due to financial constraints over the past 12 months (online Supplemental Table S2). Total scores range from 0 to 6 , which indicate levels of food security. For this study, food security status was dichotomised as food secure, with less than two affirmative answers, or food insecure, with two or more affirmative answers.

BMI was measured via a basic anthropometric assessment including only height and weight. Trained study staff measured height and weight. Height was measured using a portable stadiometer, and weight was measured using a digital scale. Measurements were taken without shoes and recorded to the nearest $0.1 \mathrm{~cm}$ and $0.1 \mathrm{~kg}$, respectively. BMI was calculated (weight $(\mathrm{kg}) /$ height $\left(\mathrm{m}^{2}\right)$ ) and used to categorise participants as having normal weight (BMI $<25 \mathrm{~kg} / \mathrm{m}^{2}$ ) or overweight/ obesity (BMI $\left.\geq 25 \mathrm{~kg} / \mathrm{m}^{2}\right)^{(37)}$.

Participants were placed into one of the four food security status and BMI category groups: (1) food secure with normal weight, (2) food insecure with normal weight,
(3) food secure with overweight/obesity and (4) food insecure with overweight/obesity. The goal was to have an equal number ( $n$ 14) of participants within each of the four groups. Further, participants were matched on race, primarily between Whites and African Americans, across each of the four groups to address potential confounding of race with both food insecurity and obesity.

\section{Psychological constructs}

Four psychological constructs were investigated in this study: (1) delay discounting, (2) grit, (3) future time perspective and (4) subjective social status. These constructs were measured via seven validated questionnaires. Table 1 presents the expected association of each psychological construct with food insecurity and body weight based upon the relevant extant literature.

Delay discounting is a bias towards smaller, immediate rewards $v$. larger, delayed rewards ${ }^{(38)}$ and was assessed via the twenty-seven-item monetary choice questionnaire ${ }^{(39)}$. This questionnaire presents participants with a set of dichotomous choices between a smaller, immediate monetary reward and a larger, delayed monetary reward. Participants who discount the value of the delayed rewards more steeply are considered to be more impulsive ${ }^{(39)}$. Further, the score for delay discounting is represented by $k$, which tends to be skewed, so a natural $\log (\ln )$ transformation is utilised to approximate a normal distribution for statistical analysis ${ }^{(40)}$. Values of $k$ range from 0.00016 (ln transformation -8.74) to 0.25 (In transformation 1.39), with higher values indicating a greater preference for smaller, immediate rewards over larger, delayed rewards.

Grit is a measure of trait-level perseverance and passion for long-term goals and was assessed using the eight-item Short Grit Scale (Grit-S) ${ }^{(41)}$. Total scores range from 1 (not at all gritty) to 5 (extremely gritty). The Grit-S has adequate internal validity and test-retest reliability in adults ${ }^{(41)}$.

Future time perspective is a comprehensive assessment of one's orientation towards the future ${ }^{(18,22)}$. Four instruments were used to assess future time perspective. The future scale of the Zimbardo Time Perspective Inventory (ZTPI) measures psychological orientation towards the

Table 1 Selected psychological mechanisms and their expected relationship with food insecurity and body weight

\begin{tabular}{|c|c|c|c|c|}
\hline Psychological construct & Definition & Questionnaire & $\begin{array}{c}\text { Food } \\
\text { insecurity }\end{array}$ & $\begin{array}{l}\text { Body } \\
\text { weigh }\end{array}$ \\
\hline Delay discounting & Impulsivity & 27-Item Monetary Choice Questionnaire & $\uparrow$ & $\uparrow$ \\
\hline Grit & Perseverance & 8-Item Short Grit Scale & $\downarrow$ & $\downarrow$ \\
\hline Future time perspective & Value of future outcomes & $\begin{array}{l}\text { Zimbardo Time Perspective Inventory - future } \\
\text { scale } \\
\text { Consideration of Future Consequences } \\
\text { Scale-14 } \\
\text { Financial planning } \\
\text { Longevity }\end{array}$ & $\downarrow$ & $\downarrow$ \\
\hline Subjective social status & $\begin{array}{l}\text { Perceived rank in social } \\
\text { hierarchy }\end{array}$ & MacAuthor Scale of Subjective Social Status & $\downarrow$ & $\downarrow$ \\
\hline
\end{tabular}


future $^{(42)}$. This scale is composed of thirteen statements with Likert-type responses ( $1=$ very untrue to $5=$ very true) with a total score range of 1.62-4.85. Higher scores indicate greater future time perspective. The future scale of the ZTPI has acceptable internal and test-retest reliability $^{(42)}$. The Consideration of Future Consequences Scale (CFCS-14) assesses how individuals consider future outcomes in choosing current behaviour ${ }^{(43,44)}$. This scale contains fourteen statements for which individuals indicate whether or not the statement is characteristic of them on a scale of 1-7. Total scores are an average of all fourteen items with a range of $1-7$. Higher scores indicate greater consideration of future consequences. The CFCS-14 has acceptable reliability and validity ${ }^{(43)}$. Participants were asked a single question to assess the time period considered for financial planning: 'In planning your, or your family's, saving and spending, which of the following time periods is more important to you and your partner, if you have one?' with the following response options: no planning ( 0 years), day to day ( 0.02 years), the next few weeks ( 0.12 years), next few months ( 0.5 years), next year ( 1.0 years), next few years ( 3.0 years), next $5-10$ years ( $7 \cdot 5$ years) and longer than 10 years $(10 \cdot 0 \text { years })^{(45,46)}$. Last, participants' subjective appraisal of longevity was also assessed via a single question: 'What do you think are the chances you will live to age 75 or more (where 0 means there is not a chance you will live to 75 or more, and 100 means you will definitely live to 75 or more)? ${ }^{\text {(45,46) }}$. Higher values for the financial planning and longevity questions indicate greater future time perspective.

Subjective social status (SSS) is one's self-perceived social position in US society and was measured using the MacArthur Scale of Subjective Social Status ${ }^{(47)}$. This scale presents a 'ladder' and asks participants to select a rung on which they feel they stand relative to other people in the US Scores range from 1 to 10 , with higher scores representing higher SSS. The MacArthur Scale of SSS is a reliable and valid measure of $\operatorname{SSS}^{(48,49)}$.

\section{Sociodemographics}

A sociodemographic survey was used to collect additional participant information, including race (White and African American), sex, age, education and income. Three participants reported their race as Asian and were grouped with White participants.

\section{Quantitative statistical analysis}

Participant characteristics were reported as stratified by food security status and BMI category. Categorical variables were reported as $n$ (\%), while continuous variables were reported as mean (SD). Simple and partial correlations, adjusted for food security status, BMI category, sex, age and income, between scores on each of the psychological questionnaires were assessed to test for significant associations between these measures given the potential inter-relatedness of these constructs. The objective of the statistical analyses was to examine $a$ priori differences in the relationships between food security status, BMI category and scores on psychological questionnaires. Linear mixed models were regressed using scores from each of the seven psychological questionnaires as dependent variables. These models included interaction terms between food security status and BMI category. Least squares means produced by the mixed models were examined to test for significant differences in psychological questionnaire scores by food security status and BMI category group. Kenward-Roger approximation was used to calculate denominator df for fixed effects tests ${ }^{(50)}$. All models included sex, age and income as covariates. $P \leq 0.05$ was used to determine significance. Data analyses were conducted using SPSS (IBM SPSS Statistics 25) and SAS (version 9.4) software.

\section{Qualitative data collection and analysis}

Following completion of quantitative data collection, all participants were asked if they were interested in participating in an in-depth interview. From those who were willing to participate in the interview, a convenience sample of twelve participants was selected for the in-depth interview. This sample size was targeted given evidence that 'saturation,' or the point at which no new information or themes are derived, is often achieved with this number of interviews ${ }^{(51)}$. Again, equal distribution between the four food security status and BMI category groups was targeted. A semi-structured interview guide with open-ended questions was developed to probe for detailed information regarding personal experiences with food security and food insecurity, body weight and health issues, and the psychological constructs measured via questionnaires administered during quantitative data collection. Following written informed consent, in-depth interviews were conducted in-person and were audio recorded given permission by the participant. Interviews were transcribed verbatim. De-identified transcripts were uploaded to qualitative data analysis software $\left(\right.$ MAXQDA $\left.^{\odot}\right)$, which was used to organise and analyse the qualitative data. A thematic analysis of textual data was undertaken using deductive codes based upon pre-determined themes from the interview guide and quantitative portion of the study. Coded textual data were then compared between and within the four food security status and BMI category groups to better understand how selected issues were discussed and subjectively perceived by these subgroups of participants ${ }^{(28)}$.

\section{Results}

\section{Quantitative results}

As shown in Table 2, an equal distribution of participants was not achieved between each of the four food security 
Table 2 Participant characteristics by food security status and BMI category*

\begin{tabular}{|c|c|c|c|c|c|c|c|c|}
\hline \multirow[b]{2}{*}{ Food security score } & \multicolumn{2}{|c|}{$\begin{array}{l}\text { Food secure with } \\
\text { normal weight } \\
(n 13)\end{array}$} & \multicolumn{2}{|c|}{$\begin{array}{l}\text { Food insecure } \\
\text { with normal } \\
\text { weight }(n 13)\end{array}$} & \multicolumn{2}{|c|}{$\begin{array}{c}\text { Food secure with } \\
\text { overweight/ } \\
\text { obesity }(n 13)\end{array}$} & \multicolumn{2}{|c|}{$\begin{array}{l}\text { Food insecure } \\
\text { with overweight/ } \\
\text { obesity }(n 17)\end{array}$} \\
\hline & 0.2 & 0.4 & 4 & 1.7 & 0.5 & 0.5 & 4.7 & 1.4 \\
\hline BMI $\left(\mathrm{kg} / \mathrm{m}^{2}\right)$ & $22 \cdot 9$ & 1.5 & $22 \cdot 0$ & 1.4 & $32 \cdot 6$ & 7.3 & 35.5 & 5.9 \\
\hline African American & 5 & 38.5 & 5 & 38.5 & 6 & $46 \cdot 2$ & 11 & 64.7 \\
\hline Female & 10 & $76 \cdot 9$ & 9 & 69.2 & 7 & 53.8 & 11 & 64.7 \\
\hline Age & $21 \cdot 8$ & $7 \cdot 6$ & $27 \cdot 6$ & $7 \cdot 7$ & 30.5 & $10 \cdot 5$ & $37 \cdot 8$ & $8 \cdot 2$ \\
\hline \multicolumn{9}{|l|}{ Education } \\
\hline Some high school & 0 & 0 & 1 & $7 \cdot 7$ & 1 & $7 \cdot 7$ & 0 & 0 \\
\hline High school diploma/GED & 3 & $23 \cdot 1$ & 3 & $23 \cdot 1$ & 6 & $46 \cdot 2$ & 8 & $47 \cdot 1$ \\
\hline Some college & 1 & $7 \cdot 7$ & 3 & $23 \cdot 1$ & 2 & $15 \cdot 4$ & 5 & 29.4 \\
\hline Bachelor's degree & 3 & $23 \cdot 1$ & 5 & 38.5 & 1 & $7 \cdot 7$ & 2 & $11 \cdot 8$ \\
\hline Graduate/professional degree & 6 & $46 \cdot 2$ & 1 & $7 \cdot 7$ & 3 & 23.1 & 2 & 11.8 \\
\hline \multicolumn{9}{|l|}{ Income (annual household) } \\
\hline$<\$ 10000$ & 0 & 0 & 4 & $30 \cdot 8$ & 2 & $15 \cdot 4$ & 9 & $52 \cdot 9$ \\
\hline$\$ 10000-\$ 29999$ & 3 & $23 \cdot 1$ & 6 & $46 \cdot 2$ & 3 & $23 \cdot 1$ & 5 & 29.4 \\
\hline$\geq \$ 30000$ & 8 & 61.5 & 3 & $23 \cdot 1$ & 7 & 53.8 & 3 & $17 \cdot 6$ \\
\hline Health literacy score & $7 \cdot 0$ & 0 & 6.7 & 0.5 & $5 \cdot 7$ & $2 \cdot 1$ & $5 \cdot 7$ & 1.8 \\
\hline$\leq 6(\leq 8$ th grade $)$ & 0 & 0 & 4 & $30 \cdot 8$ & 56 & 38.5 & 8 & $47 \cdot 1$ \\
\hline$\overline{7}$ ( $\geq 9$ th grade) & 13 & $100 \cdot 0$ & 9 & 69.2 & 8 & 61.5 & 9 & 52.9 \\
\hline \multicolumn{9}{|l|}{ Psychological Questionnaires } \\
\hline Delay discounting $(\ln (\mathrm{k}))$ & $-5 \cdot 1$ & $1 \cdot 8$ & $-5 \cdot 1$ & 1.9 & $-5 \cdot 2$ & $2 \cdot 8$ & $-4 \cdot 1$ & 1.8 \\
\hline Grit & 3.9 & 0.4 & 3.4 & 0.6 & 3.9 & 0.7 & 3.5 & 0.7 \\
\hline ZTPI - future scale & 3.7 & 0.2 & 3.7 & 0.3 & 3.5 & 0.6 & 3.9 & 0.6 \\
\hline CFCS-14 & $5 \cdot 4$ & 0.6 & $5 \cdot 1$ & 0.9 & $5 \cdot 1$ & 0.7 & $5 \cdot 0$ & 0.9 \\
\hline Financial planning (years) & $5 \cdot 1$ & 4.0 & 1.9 & $3 \cdot 2$ & $2 \cdot 2$ & 4.0 & 0.9 & 2.5 \\
\hline Longevity & $91 \cdot 2$ & $16 \cdot 7$ & $84 \cdot 2$ & $13 \cdot 2$ & 76.5 & 37.6 & $93 \cdot 8$ & $10 \cdot 4$ \\
\hline SSS & $5 \cdot 8$ & 1.7 & 5.2 & 1.2 & 5.9 & 2.2 & 4.5 & 2.3 \\
\hline
\end{tabular}

ZTPI, Zimbardo Time Perspective Inventory; CFCS-14, Consideration of Future Consequences Scale; SSS, subjective social status.

${ }^{*}$ Categorical variables reported as $n$ and \%. Continuous variables reported as mean and standard deviation.

Table 3 Simple and partial correlations between psychological mechanisms $†$

\begin{tabular}{|c|c|c|c|c|c|c|c|}
\hline & 1 & 2 & 3 & 4 & 5 & 6 & 7 \\
\hline 1. Delay discounting $(\ln (\mathrm{k}))$ & - & -0.119 & -0.151 & -0.012 & -0.152 & -0.079 & -0.196 \\
\hline 2. Grit & -0.116 & - & 0.139 & $0.371^{*}$ & 0.203 & 0.136 & $-0.410^{* *}$ \\
\hline 3. ZTPI - future scale & -0.188 & 0.069 & - & 0.092 & 0.046 & $0.494^{\star \star *}$ & 0.208 \\
\hline 4. CFCS-14 & 0.010 & $0.280^{*}$ & 0.089 & - & 0.089 & 0.147 & -0.242 \\
\hline 5. Financial planning (years) & -0.136 & 0.188 & -0.023 & 0.223 & - & 0.219 & 0.068 \\
\hline 6. Longevity & 0.102 & -0.139 & $0.431^{\star * *}$ & 0.210 & 0.130 & - & 0.137 \\
\hline 7. SSS & 0.112 & -0.174 & -0.039 & -0.041 & 0.188 & 0.088 & - \\
\hline
\end{tabular}

ZTPI, Zimbardo Time Perspective Inventory; CFCS-14, Consideration of Future Consequences Scale; SSS, subjective social status.

†Simple correlations below diagonal. Partial correlations (adjusted for food security status, BMI category, sex, age, and income) above diagonal.

${ }^{*} P<0.05,{ }^{* *} P<0.01,{ }^{* * *} P<0.001$.

and BMI category groups, with a greater number of participants in the food-insecure and overweight/obese category. As expected, food security scores and BMI were significantly different between groups $(P<0 \cdot 001)$. There were no significant differences in the percentage of female participants $(P=0.65)$ or educational attainment $(P=0 \cdot 19)$. However, there were significant differences in age $(P=0.011)$ and income $(P=0.016)$. Food-insecure participants with overweight/obesity were on average older compared with the other three groups. A greater percentage of food-secure participants with normal weight reported an annual household income greater than or equal to $\$ 30000$, while a greater percentage of food-insecure participants with overweight/obesity reported an annual household income less than $\$ 10000$.
Table 3 shows the results of the correlation analyses. There was a significant and positive simple correlation between scores on the CFCS-14, an indicator of future time perspective, and grit $(r=0 \cdot 28 ; P=0 \cdot 04)$, which was similar to the respective partial correlation for these two constructs $(r=0.37, P=0.02)$. Self-reported longevity was significantly and positively correlated with ZTPI $(r=0.43$, $P=0.001$ ), both of which are indicators of future time perspective. This correlation remained significant with the adjustment of multiple covariates $(r=0.49, P=0.001)$. SSS shared a significant and negative partial correlation with grit $(r=-0 \cdot 41, P=0 \cdot 01)$.

Figure 1 shows results from linear mixed models testing for differences in delay discounting (a), grit (b), ZTPI (c), CFCS-14 (d), financial planning (e), longevity (f) and 
(a)

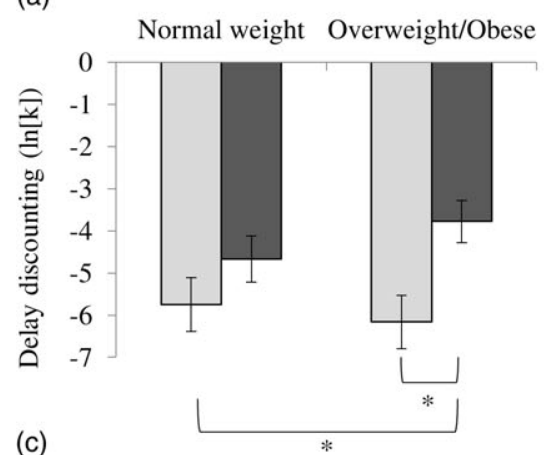

(c)

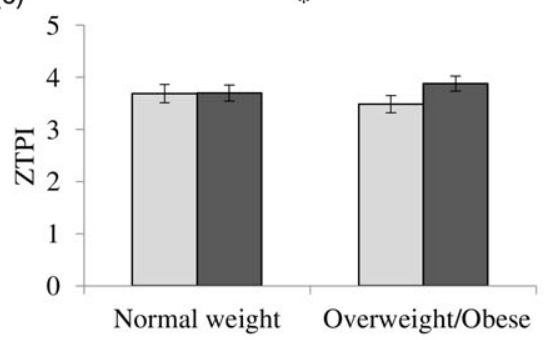

(e)

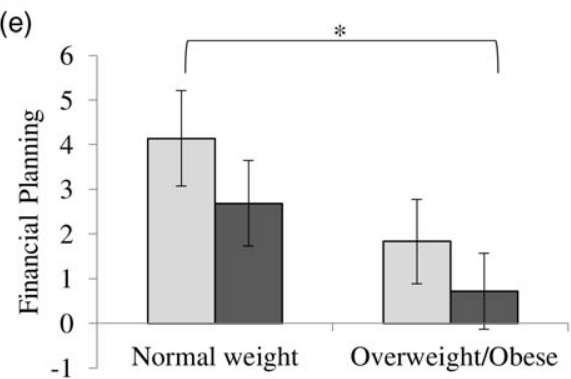

(b)

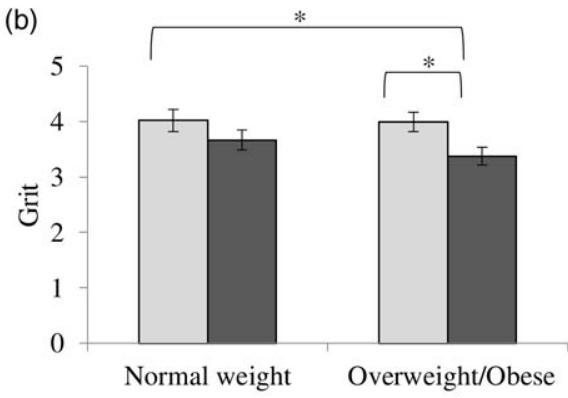

(d)

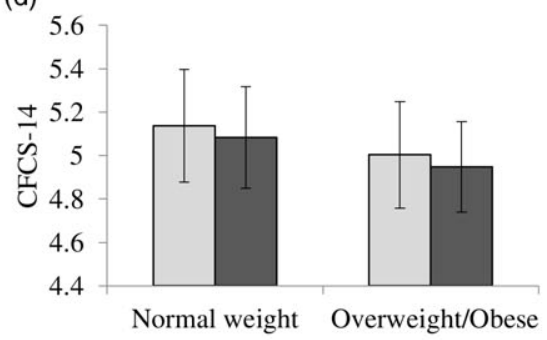

(f)

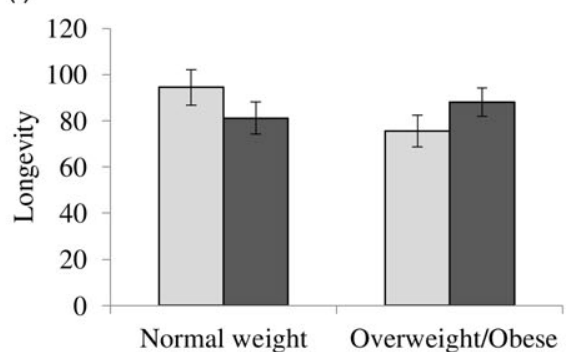

(g)

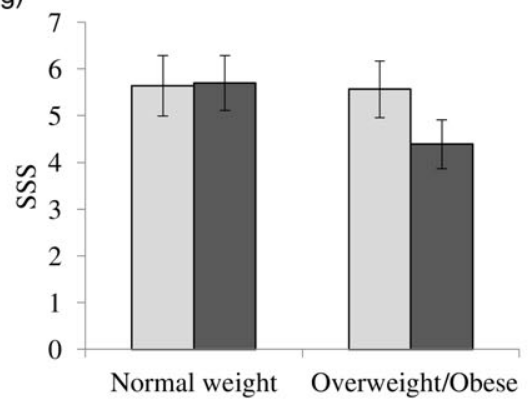

Fig. 1 Adjusted means of Psychological Questionnaire Scores by food security status and BMl category. (a) Significant differences in delay discounting between food-insecure adults with overweight/obesity and (1) food-secure adults with overweight/obesity and (2) food-secure adults with normal weight. (b) Significant differences in grit between food-insecure adults with overweight/obesity and (1) food-secure adults with overweight/obesity and (2) food-secure adults with normal weight. (e) Significant difference in the time period for financial planning between food-insecure adults with overweight/obesity and food-secure adults with normal weight. No significant differences in (c) Zimbardo Time Perspective Inventory (ZTPI), (d) Consideration of Future Consequences Scale (CFCS)-14, (f) subjective longevity and (g) subjective social status (SSS) between food security status and BMI category groups. ${ }^{\star} P<0.05$. $\square$, Food secure; $\square$, Food insecure

SSS (g) between food security and BMI category groups. Results show that food-insecure participants with overweight/obesity had significantly greater delay discounting (a) than food-secure counterparts $(-3.78 v$. $-6 \cdot 16, P=0 \cdot 01)$, as well as when compared with foodsecure participants with normal weight $(-3.78 v .-5 \cdot 75$, $P=0.02)$. Food-insecure participants with overweight/ obesity also had significantly less grit (b) than food-secure counterparts (3.37 v. 3.99, $P=0.02)$ and food-secure participants with normal weight $(3.37 v$. 4.02, $P=0.02$ ). Food-insecure participants with overweight/obesity had a significantly smaller timeframe for financial planning (e) compared with food-secure participants with normal weight $(0.72 v .4 \cdot 14, P=0 \cdot 02)$. There were no significant differences in ZTPI (c), CFCS-14 (c), longevity (f) and SSS (g). 


\section{Qualitative results}

Descriptive characteristics are provided in online Supplemental Table S1 for those participants that underwent the in-depth interview. Results are presented under three overarching themes, which were the focus of this study: food security status, body weight and psychological mechanisms.

\section{Food security status}

When asked about their understanding of both food security and food insecurity, most participants, regardless of their own food security status or BMI category, provided similar definitions. Specifically, participants noted issues related to 'capital' or 'financial resources' that either allowed or constrained the ability to 'afford' food. Participants further noted the quality of food, such as 'nutritious,' 'healthy' or 'fresh.' Interestingly, one food-secure participant noted that food insecurity could lead to the need to 'even dig in the garbage can.' This food-seeking strategy was actually documented by a food-insecure participant who detailed previous experiences of having 'to eat out of dumpsters to feed my kids or I would have to beg people to feed them.' Such experiences that result from food insecurity are important as the definition of food insecurity does denote the inability 'to acquire acceptable foods in socially accept-

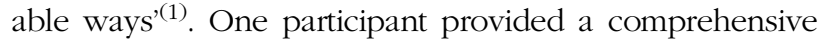
description of food security that captured a number of resources:

'I guess just having...uh, consistent work... and access to cars ... and ... uh, you know grocery stores and the means to prepare the food. So, all of that I guess would be food security.'

\section{Body weight}

Concerning body weight and other health issues as related to one's food security status, one participant with normal weight noted that being food insecure impacted their ability to be physically active and exercise:

'I'd say it can negatively impact my ability to be active, because sometimes since I don't have enough money I try to pick up more and more [work] shifts. So I don't have as much free time or leisure to do more activities as opposed to just be like at work sitting.'

Another food-secure participant with overweight/obesity noted that it was easy to overeat given that their family always had enough money to afford food. This experience was echoed by another food-secure participant with overweight/obesity as they noted that their family income allowed them to make 'bad choices' concerning their diet, including going out to eat, eating red meat or just eating whatever they want. A number of food-insecure participants noted that financial constraints resulted in unhealthy eating, which in turn, from their perspective, led to weight gain, as well as other health issues, such as high cholesterol or high blood pressure. Specifically, some participants noted that their struggles with food insecurity made it difficult to manage these health conditions via a healthy diet and that such issues were often exacerbated or not addressed due to constrained dietary options or purchasing 'cheaper' foods.

\section{Psychological constructs}

Many participants noted the relevance of the discussed psychological constructs when thinking about their food security status and body weight. One food-secure participant with normal weight noted that they:

'delay gratification all day when I choose healthier foods. You know, that's my delayed gratification that directly, you know, relates back to my weight.'

However, a food-insecure participant with normal weight noted that delaying immediate gratification was difficult:

'because like a lot of times when it comes to having money right now, I may need it right now, so sometimes like delaying that gratification is not my priority.'

Another food-insecure participant with normal weight related delaying gratification to having food. Specifically:

'if you don't have any food in the refrigerator then there is no sacrifice. There is no compromise.'

In relation to quantitative data, these insights from participants demonstrate that those who were food secure with normal weight reported the perceived importance of delaying gratification in order to maintain a healthy body weight. However, those who were food insecure struggled with delaying gratification in the face of financial constraints and reported that a lack of money created unintentional delaying of gratification when there was no food available.

Concerning grit, one food-secure participant with normal weight noted that they perceived their household to have a lot of grit or passion for long-term goals. However, this psychological strength was not of consequence 'because we have the financial resources that we don't need to use that.' This was also noted by another food-secure participant with overweight/obesity as their household always had enough to eat, so this was not a challenge because their finances afforded the ability to always purchase food to eat. Another food-secure participant with normal weight also expressed that grit was not an important consideration for them given their current 'metabolism,' which they felt allowed them to not worry about managing their body weight. However, a food-insecure participant with normal weight did feel that grit was important when considering their body weight. This participant cited that taking on extra work for additional income and 'the tenacity of trying to make sure we have enough, going the extra 
mile, and [working] overtime' to make sure there were sufficient financial resources to support a healthy diet. This was further echoed by another food-insecure participant with normal weight who found that managing their body weight was achievable via 'small goals to reach big goals and grit plays a huge part in that.' For those foodinsecure participants with overweight/obesity, grit was not discussed in-depth or often considered by these individuals. Compared with quantitative results, which showed significant differences in grit, the qualitative insights provided by participants showed that grit was largely important to those with normal weight when considering both their food security status and managing their body weight. Additionally, food-secure participants, regardless of weight status, reported that grit was not as important in the context of financial security.

Regarding perceiving and planning for the future, a food-secure participant with normal weight reported that they planned for a 'lifetime' and were 'lucky that we can plan for the future, because we have enough money.' In contrast, a food-insecure participant with normal weight noted that they plan 'basically month to month' and that it further:

'depends on the time of the year, because it's like now we be in kind of a little excess because of daylight savings time. That gives me more time in the evening to go cut more grass and stuff. And I enjoy it, but it brings more to the table, brings more income to the table.'

It was also noted that it was sometimes difficult to plan for the future if 'unfortunate things happen,' such as a car breaking down or if a family member gets sick and requires medical treatment, which was reported to impact or compromise the food budget. One food-secure participant with overweight/obesity noted a time of being food insecure during college and recalled that their experience with perceiving the future was different during that time:

'I wasn't making the best decisions on a daily basis and so, also you're too busy worrying about surviving in that moment to think about the future definitely. And also then you're thinking about how to get by day to day, you're not thinking about... you're not planning meals and so you'll grab [fast food] or whatever.'

Among food-insecure participants with overweight/ obesity, thinking about the future was limited compared with other participants. One participant noted that they:

'don't plan for their life. I plan with my resources for month to month ... You know like today I don't have any money. You know I might walk out the door and I got a lot of money. So, no, I don't like making plans.'

A similar participant noted that 'we rarely get a chance to save anything.' Additionally, another food-insecure participant recalled a time that their financial situation improved for a short time after receiving a large payout following a car accident. Quantitative results also showed significant differences in financial planning. Qualitatively, foodinsecure participants, regardless of weight status, largely noted that the period considered for financial planning was much shorter than that considered by those who were food secure.

Regarding subjective social status, most participants felt it was important. One food-secure participant with normal weight noted that 'depending on social status, you get more or better services' and also related social status to having a good job, which allows for:

'you to be able to afford stuff and you just feel better and more comfortable and you go about your life with an ease.'

Another food-insecure participant was cognizant of their presentation of self when it came to social status:

'I may not have the income, but a lot of people don't realize that. You know, they might look at me and say 'man, this guy must make like $\$ 50000$ or $\$ 60000$ a year' and I don't you know, but it's how I present myself.'

Social status was also related to stress and mental health for a number of food-insecure participants. One participant noted that social status:

'raises your self-image and how you feel about yourself. And, I feel like that also plays a big deal in your health as well, because I mean it's just the stress of thinking about it.'

Another food-insecure participant noted that considering their social status impacted their mental health due to stress and worrying about providing both necessities and luxuries for their family, specifically clothing items that may be held in high regard by others. This stress, in turn, was identified as having an impact on the participant's eating behaviour and body weight because:

'sometimes you feel like you are eating more than you need to and then sometimes you are feeling like you are eating less than you need to.'

While quantitative results did not indicate significant differences in SSS, these perceptions reported by participants show that regardless of food security status or body weight, most believed that social status was a relevant issue.

\section{Discussion}

This mixed methods study investigated decision-making psychological mechanisms in relation to food security status and body weight in adults with the purpose of identifying new factors that play a role in the relationship between food insecurity and obesity. Specifically, delay discounting, grit, future time perspective and subjective 
social status were measured in a sample of food-secure and food-insecure adults with normal weight and overweight/ obesity. Quantitative results found that food-insecure individuals with overweight/obesity had poorer delay discounting and grit than both food-secure counterparts and food-secure individuals with normal weight. It was further shown that the timeframe considered for financial planning was much smaller for food-insecure individuals with overweight/obesity compared with food-secure individuals with normal weight. Qualitative data from in-depth interviews provided further insight into the complex interplay of food security status and body weight by highlighting differential perceptions of the psychological constructs measured in this study.

The results herein provide support for the insurance hypothesis. While Nettle et $\mathrm{al}^{(2)}$ did not designate explicit decision-making mechanisms, this study drew from the literature to identify psychological constructs that held potential influence. Results demonstrated the importance of delay discounting, grit and the time period of financial planning as a marker of future time perspective as important psychological mechanisms to be considered in the food insecurity-obesity linkage. Other obesity researchers have noted the importance of delay discounting as a psychosocial construct that should be a standardised assessment in obesity studies and treatment programmes ${ }^{(52)}$. In this study, delay discounting was noted to be important for maintaining a healthy body weight, yet others discussed that being food insecure compromised the ability to delay gratification. Grit was also associated with managing body weight and was further said to be important for securing financial stability. Financial planning emerged as the most relevant aspect of future time perspective, which is logical given that food security status is strongly linked to income and other financial resources. Those participants who reported being food insecure discussed that their financial planning timeline was much smaller (i.e. 'month to month') compared with those who were food secure (i.e. 'a lifetime'). Last, subjective social status did not emerge as a quantitatively significant decisionmaking construct, but did elicit-rich qualitative feedback from most participants. This does provide some support for the resource scarcity hypothesis and its focus on social status as a mechanistic component in the food insecurityobesity linkage ${ }^{(15)}$

Strengths of this study include the use of a mixed methods approach. The qualitative information gathered from participants provided unique contextual data and subjective insights to complement findings from quantitative analyses. Secondly, the community partnership formed with a local food pantry for this study allowed for the targeted recruitment of food-insecure participants. Despite these strengths, a number of study limitations are noted. The first includes the cross-sectional nature of this study, which limits the ability to infer causal directionality in the food insecurity-obesity linkage. Further, the participant population in this study was largely White and African American, with little representation from other racial and ethnic minority groups. This limits the generalisability of study findings to other population subgroups. In addition, the sample size for this study was relatively small and was divided further into four groups based upon food security status and BMI category. The small sample size may potentially drive the significant or non-significant effects shown, and results from this study should be interpreted with caution given that reliability may be limited. However, future studies should undertake similar investigations in larger samples to provide further evidence into the relationships examined in this study. Last, this study only focused on a single nutritional risk factor, food security status, in relation to body weight. It is important to recognise that obesity is the result of multiple factors related to energy intake and energy expenditure. This study did not assess diet and physical activity, or the contextual environments (e.g. neighbourhood or community) in which individuals reside, work or commute. Given this, this study does not address the potential energy intake, energy expenditure and environmental factors that may be relevant in understanding why food insecurity and obesity are linked. Certainly, future studies should collect such data concurrently with psychological assessments to create a comprehensive picture of the pathway linking food insecurity to obesity.

\section{Conclusions}

This study integrated new hypotheses and diffuse empirical evidence to investigate multiple psychological mechanisms as potential factors underlying the association between food insecurity and obesity. Evidence shown here suggests a number of psychological mechanisms, including delay discounting, grit and financial planning, are relevant to understanding the food insecurity-obesity linkage. This evidence further suggests the need to explore the modifiability of these mechanisms in the context of behaviour change interventions targeting health improvement. That is, obesity treatment in low-income populations facing food insecurity may benefit from targeting and modifying psychological mechanisms in addition to standard energy balance behaviours.

\section{Acknowledgements}

Acknowledgements: The authors would like to thank Theresa Sandifer, founder and director of The Shepherd's Market, for her partnership in this project. Financial support: This work was supported by the National Institute of General Medical Sciences of the National Institutes of Health, which funds the Louisiana Clinical and Translational Science Center (U54 GM104940) and a NORC Center Grant (no. P30DK072476) entitled 'Nutrition and Metabolic Health 
Through the Lifespan' sponsored by NIDDK. The content of this manuscript is solely the responsibility of the authors and does not necessarily represent the official views of the National Institutes of Health. Funding sources had no involvement in the design of this study; in the collection, analysis and interpretation of data; in the writing of the report; and in the decision to submit the article for publication. Conflict of interest: The authors have no conflicts of interest in relation to this work. Authorship: C.A.M. made substantial contributions to the conception and design of the study, data acquisition, and analysis and interpretation of the data; drafted the article and revised critically for important intellectual content; and gave final approval for this version of the manuscript. R.A.B. made substantial contributions to the conception and design of the study and analysis and interpretation of the data; critically revised the manuscript for important intellectual content; and gave final approval for this version of the manuscript. C.K.M. made substantial contributions to the conception and design of the study; critically revised the manuscript for important intellectual content; and gave final approval for this version of the manuscript. S.T.B. made substantial contributions to the conception and design of the study; critically revised the manuscript for important intellectual content; and gave final approval for this version of the manuscript. P.T.K. made substantial contributions to the conception and design of the study and analysis and interpretation of the data; critically revised the manuscript for important intellectual content; and gave final approval for this version of the manuscript. Ethics of buman subject participation: This study was conducted according to the guidelines laid down in the Declaration of Helsinki, and all procedures involving study participants were approved by the Pennington Biomedical Research Center (PBRC) Institutional Review Board. Written informed consent was obtained from all participants. This study was also registered at ClinicalTrials.gov (Identifier NCT03441594).

\section{Supplementary material}

For supplementary material accompanying this paper visit https://doi.org/10.1017/S1368980020000889

\section{References}

1. Anderson SA (1990) Core indicators of nutritional state for difficult-to-sample populations. J Nutr 120, Suppl. 11, 1559-1600.

2. Nettle D, Andrews C \& Bateson M (2016) Food insecurity as a driver of obesity in humans: the insurance hypothesis. Behav Brain Sci 40, 1-34.

3. Laraia BA (2013) Food insecurity and chronic disease. Adv Nutr 4, 203-212.

4. Seligman HK \& Schillinger D (2010) Hunger and socioeconomic disparities in chronic disease. $N$ Engl J Med 363, 6-9.
5. Townsend MS, Peerson J, Love B et al. (2001) Food insecurity is positively related to overweight in women. J Nutr 131, 1738-1745.

6. Drewnowski A \& Specter SE (2004) Poverty and obesity: the role of energy density and energy costs. Am J Clin Nutr 79, 6-16.

7. Leung CW \& Tester JM (2018) The association between food insecurity and diet quality varies by race/ethnicity: an analysis of national health and nutrition examination survey 20112014 results. J Acad Nutr Diet 199, 1676-1686.

8. Stinson EJ, Votruba SB, Venti C et al. (2018) Food insecurity is associated with maladaptive eating behaviors and objectively measured overeating. Obesity 26, 1841-1848.

9. Wu CH, Lin CY, Hsieh YP et al. (2019) Dietary behaviors mediate the association between food insecurity and obesity among socioeconomically disadvantaged youth. Appetite 132, 275-281.

10. Dietz WH (1995) Does hunger cause obesity? Pediatrics 95 $766-767$.

11. Adams EJ, Grummer-Strawn L \& Chavez G (2003) Food insecurity is associated with increased risk of obesity in California women. J Nutr 133, 1070-1074.

12. Dinour LM, Bergen D \& Yeh MC (2007) The food insecurityobesity paradox: a review of the literature and the role food stamps may play. J Am Diet Assoc 107, 1952-1961.

13. Castillo DC, Ramsey NL, Yu SS et al. (2012) Inconsistent access to food and cardiometabolic disease: the effect of food insecurity. Curr Cardiovasc Risk Rep 6, 245-250.

14. Saiz AM Jr, Aul AM, Malecki KM et al. (2016) Food insecurity and cardiovascular health: findings from a statewide population health survey in Wisconsin. Prev Med 93, 1-6.

15. Dhurandhar EJ (2016) The food-insecurity obesity paradox: a resource scarcity hypothesis. Physiol Behav 162, 88-92.

16. Laraia BA, Leak TM, Tester JM et al. (2017) Biobehavioral factors that shape nutrition in low-income populations: a narrative review. Am J Prev Med 52, Suppl. 2, S118-S126.

17. Epstein LH, Jankowiak N, Fletcher KD et al. (2014) Women who are motivated to eat and discount the future are more obese. Obesity 22, 1394-1399.

18. Adams J \& Nettle D (2009) Time perspective, personality and smoking, body mass, and physical activity: an empirical study. Br J Health Psychol 14, 83-105.

19. Graham Thomas J, Seiden A, Koffarnus MN et al. (2015) Delayed reward discounting and grit in men and women with and without obesity. Obes Sci Pract 1, 131-1315.

20. Mazur JE (1987) An adjusting procedure for studying delayed reinforcement. In The Effect of Delay and of Intervening Events on Reinforcement Value. Quantitative Analyses of Behavior, vol. 5, pp. 55-73. Hillsdale, NJ: Lawrence Erlbaum Associates, Inc.

21. Duckworth AL, Peterson C, Matthews MD et al. (2007) Grit: perseverance and passion for long-term goals. J Pers Soc Psychol 92, 1087-1101.

22. Epstein LH, Jankowiak N, Lin H et al. (2014) No food for thought: moderating effects of delay discounting and future time perspective on the relation between income and food insecurity. Am J Clin Nutr 100, 884-890.

23. Nikolaus CJ, Schierer M, Ellison B et al. (2019) Grit is associated with food security among US parents and adolescents. Am J Health Behav 43, 207-218.

24. Dhurandhar EJ, Pavela G, Kaiser KA et al. (2018) Body mass index and subjective social status: the coronary artery risk development in young adults study. Obesity 26, 426-431.

25. Willis DE \& Fitzpatrick KM (2016) Psychosocial factors as mediators of food insecurity and weight status among middle school students. Appetite 103, 236-243.

26. Morse JM (1991) Approaches to qualitative-quantitative methodological triangulation. Nurs Res 40, 120-123.

27. Fetters MD, Curry LA \& Creswell JW (2013) Achieving integration in mixed methods designs-principles and practices. Health Serv Res 48, 2134-2156. 
28. Hennink M, Hutter I \& Bailey A (2011) Qualitative Research Methods. London: Sage.

29. FAO, IFAD, UNICEF et al. (2019) The State of Food Security and Nutrition in the World 2019: Safeguarding Against Economic Slowdowns and Downturns. Rome: FAO.

30. Amarya S, Singh K \& Sabharwal M (2015) Changes during aging and their association with malnutrition. J Clin Gerontol Geriatr 6, 78-84.

31. Arozullah AM, Yarnold PR, Bennett CL et al. (2007) Development and validation of a short-form, rapid estimate of adult literacy in medicine. Med Care 45, 1026-1033.

32. Harris PA, Taylor R, Thielke R et al. (2009) Research electronic data capture (REDCap) - a metadata-driven methodology and workflow process for providing translational research informatics support. J Biomed Inform 42, 377-381.

33. Harris PA, Taylor R, Minor BL et al. (2019) The REDCap consortium: building an international community of software platform partners. J Biomed Inform 95, 103208.

34. Seligman HK, Davis TC, Schillinger D et al. (2010) Food insecurity is associated with hypoglycemia and poor diabetes self-management in a low-income sample with diabetes. $J$ Health Care Poor Underserved 21, 1227-1233.

35. Bickel G, Nord M, Price C et al. (2000) Guide to Measuring Household Food Insecurity. Alexandria, VA: Agriculture USDo, Service FaN, Office of Analysis N, and Evaluation.

36. Blumberg SJ, Bialostosky K, Hamilton WL et al. (1999) The effectiveness of a short form of the Household Food Security Scale. Am J Public Health 89, 1231-1234.

37. World Health Organization (WHO) (2018) Obesity and Overweight. https://www.who.int/news-room/fact-sheets/ detail/obesity-and-overweight (accessed February 2020).

38. Sze YY, Stein JS, Bickel WK et al. (2017) Bleak present, bright future: online episodic future thinking, scarcity, delay discounting, and food demand. Clin Psychol Sci 5, 683-697.

39. Kirby KN, Petry NM \& Bickel WK (1999) Heroin addicts have higher discount rates for delayed rewards than non-drug-using controls. J Exp Psychol Gen 128, 78-87.

40. Kaplan BA, Amlung M, Reed DD et al. (2016) Automating scoring of delay discounting for the 21- and 27-item monetary choice questionnaires. Behav Anal 39, 293-304.
41. Duckworth AL \& Quinn PD (2009) Development and validation of the short grit scale (grit-s). J Pers Assess 91, 166-174.

42. Zimbardo PG \& Boyd JN (1999) Putting time in perspective: a valid, reliable individual-differences metric. J Pers SOC Psychol 77, 1271-1288.

43. Strathman A, Gleicher F, Boninger DS et al. (1994) The consideration of future consequences: weighing immediate and distant outcomes of behavior. JPers Soc Psychol 66, 742-752.

44. Joireman J, Shaffer MJ, Balliet D et al. (2012) Promotion orientation explains why future-oriented people exercise and eat healthy: evidence from the two-factor consideration of future consequences-14 scale. Pers Soc Psychol Bull 38, $1272-1287$.

45. Nagin DS \& Pogarsky G (2004) Time and punishment: delayed consequences and criminal behavior. J Quant Criminol 20, 295-317.

46. Picone G, Sloan F \& Taylor D Jr (2004) Effects of risk and time preference and expected longevity on demand for medical tests. J Risk Uncertain 28, 39-53.

47. Adler NE, Epel ES, Castellazzo G et al. (2000) Relationship of subjective and objective social status with psychological and physiological functioning: preliminary data in healthy white women. Health Psychol 19, 586-592.

48. Giatti L, Camelo Ldo V \& Rodrigues JF et al. (2012) Reliability of the MacArthur scale of subjective social status - Brazilian Longitudinal Study of Adult Health (ELSA-Brasil). BMC Public Health 12, 1096.

49. Ferreira WA, Giatti L, Figueiredo RC et al. (2018) Concurrent and face validity of the MacArthur scale for assessing subjective social status: Brazilian Longitudinal Study of Adult Health (ELSA-Brasil). Cien Saude Colet 23, 1267-1280.

50. Kenward MG \& Roger JH (1997) Small sample inference for fixed effects from restricted maximum likelihood. Biometrics 53, 983-997.

51. Guest G, Bunce A \& Johnson L (2006) How many interviews are enough? An experiment with data saturation and variability. Field Methods 18, 59-82.

52. Sutin AR, Boutelle K, Czajkowski SM et al. (2018) Accumulating Data to Optimally Predict Obesity Treatment (ADOPT) core measures: psychosocial domain. Obesity 26, Suppl. 2, S45-S54. 